\title{
Clinical Study and Review of Articles (Korean) about Retrorectal Developmental Cysts in Adults
}

\author{
Sung Wook Baek, Haeng Ji Kang', Ji Yong Yoon², Do Youn Whang, Duk Hoon Park, Seo Gue Yoon, \\ Hyun Sik Kim, Jong Kyun Lee, Jung Dal Lee ${ }^{1}$, Kwang Yun Kim \\ Departments of Surgery, ${ }^{1}$ Pathology, and ${ }^{2}$ Radiology, Seoul Song Do Colorectal Hospital, Seoul, Korea
}

Purpose: A retrorectal developmental cyst (tailgut cyst, epidermoid cyst, dermoid cyst, teratoma, and duplication) is very rare disease, and the symptoms are not characteristic so that sometimes this disease is still misdiagnosed as a supralevator abscess or a complex anal fistula. We would like to present a clinical approach to this disease.

Methods: We retrospectively examined the charts of 15 patients who were treated for retrorectal cysts from January 2001 to November 2009.

Results: All 15 patients were female. The average age was 41 years (range, 21 to 60 years). Fourteen patients (93.3\%) were symptomatic, and the most common symptom was anal pain or discomfort. Nine patients $(60 \%)$ had more than one previous operation (range, 1 to 9 times) for a supralevator abscess, an anal fistula, etc. In 12 patients (80\%), the diagnosis could be made by using the medical history and physical examination. Thirteen cysts $(80 \%)$ were excised completely through the posterior approach. The average diameter of the cysts was $4.8 \mathrm{~cm}$ (range, 2 to $10 \mathrm{~cm}$ ). Pathologic diagnoses were 8 tailgut cysts (53.3\%), 5 epidermoid cysts (33.3\%) and 2 dermoid cysts (13.3\%). The average follow-up period was 18.3 months (range, 1 to 64 months).

Conclusion: In our experience, high suspicion and physical examination are the most important diagnostic methods. If a female patient has a history of multiple perianal operations, a retrorectal bulging soft mass, a posterior anal dimple, and no conventional creamy foul odorous pus in drainage, the possibility of a retrorectal developmental cyst must be considered.

Keywords: Retrorectal tumor; Tailgut cyst; Epidermoid cyst; Dermoid cyst

\section{INTRODUCTION}

Among presacal or retrorectal developmental cysts are tailgut cysts, epidermoid cysts, dermoid cysts, teratomas and rectal intussusceptions [1-5]. (Some authors do not include teratomas and rectal intussusceptions as developmental cyst [2].) These are extremely rare types of diseases and are asymptomatic in $26-50 \%$ of the patients $[1,3,6]$. Even when a patient presents with symptoms, un-

Received: February 18, 2011 Accepted: September 8, 2011

Correspondence to: Sung Wook Baek, M.D.

Department of Colon and Rectal Surgery, Seoul Song Do Colorectal Hospital, 346-144 Shindang-3-dong, Jung-gu, Seoul 100-453, Korea

Tel: +82-2-2250-7368, Fax: +82-2-2233-8528

E-mail: baek810@naver.com

(C) 2011 The Korean Society of Coloproctology

This is an open-access article distributed under the terms of the Creative Commons Attribution NonCommercial License (http://creativecommons.org/licenses/by-nc/3.0) which permits unrestricted noncommercial use, distribution, and reproduction in any medium, provided the original work is properly cited. less the possibility of the disease is strongly suspected, the disease can be easily misdiagnosed because the presented symptoms are similar to those of inflammatory diseases, such as anorectal abscesses, complicated fistulas, and pilonidal disease [4]. If a patient undergoes inappropriate surgery, the disease will be more challenging to diagnose correctly due to structural changes and recurrence, and the fundamental treatment via complete excision will be more difficult even after the patient obtains a correct diagnosis. Also, severe complications, such as fecal incontinence or malignancy, can be induced. Thus, the first doctor who examines the patient has the best opportunity to treat the disease [7]. Especially, colorectal specialists, who mainly contact the patients with benign diseases in their private clinics, are less likely to have experience with this disease, and even when they come across a patient with a developmental cyst, they might consider perianal inflammatory disease first and perform a surgery that will make treating the disease more difficult in the future. Hence, it is important to consider the disease as a differential diagnosis. 
The authors examined the clinical progress of 15 adult patients with retrorectal developmental cysts and reviewed the literature. This study aimed to provide a proper clinical approach to the diagnosis and the treatment of retrorectal developmental cysts.

\section{METHODS}

The subjects were 15 patients who were treated for retrorectal developmental cysts in the colorectal surgery clinic in this hospital between January 2001 and November 2009. A retrospective medical record review was performed and we considered sex, age, surgical history for the same lesion, presented symptoms at the time of the diagnosis, signs that aided the diagnosis and surgical methods after the diagnosis.

\section{RESULTS}

A total of 15 patients were diagnosed with a retrorectal developmental cyst in this hospital between January 2001 and November 2009 (Tables 1 and 2). They were all female patients, and the mean age at the time of the diagnosis was 41 years old (range, 21 to 60 years old). There were 5 patient in their 20's (33.3\%), one patient in her 30's (6.6\%), 3 patients in their 40's (20\%), 5 patients in their 50 's (33.3\%), and one patient in her 60's (6.7\%).

Among the 15 patients, 14 (93.3\%) presented with symptoms and the other patient (6.7\%) was asymptomatic. In the latter patient, the cyst was found accidentally on computed tomography (CT). The most common symptoms were anal pain or perianal discomfort (53.3\%), followed by a heavy feeling in the behind (33.3\%), disturbed evacuation (20\%), perianal discharge (13.3\%), bleeding

Table 1. Patient characteristics

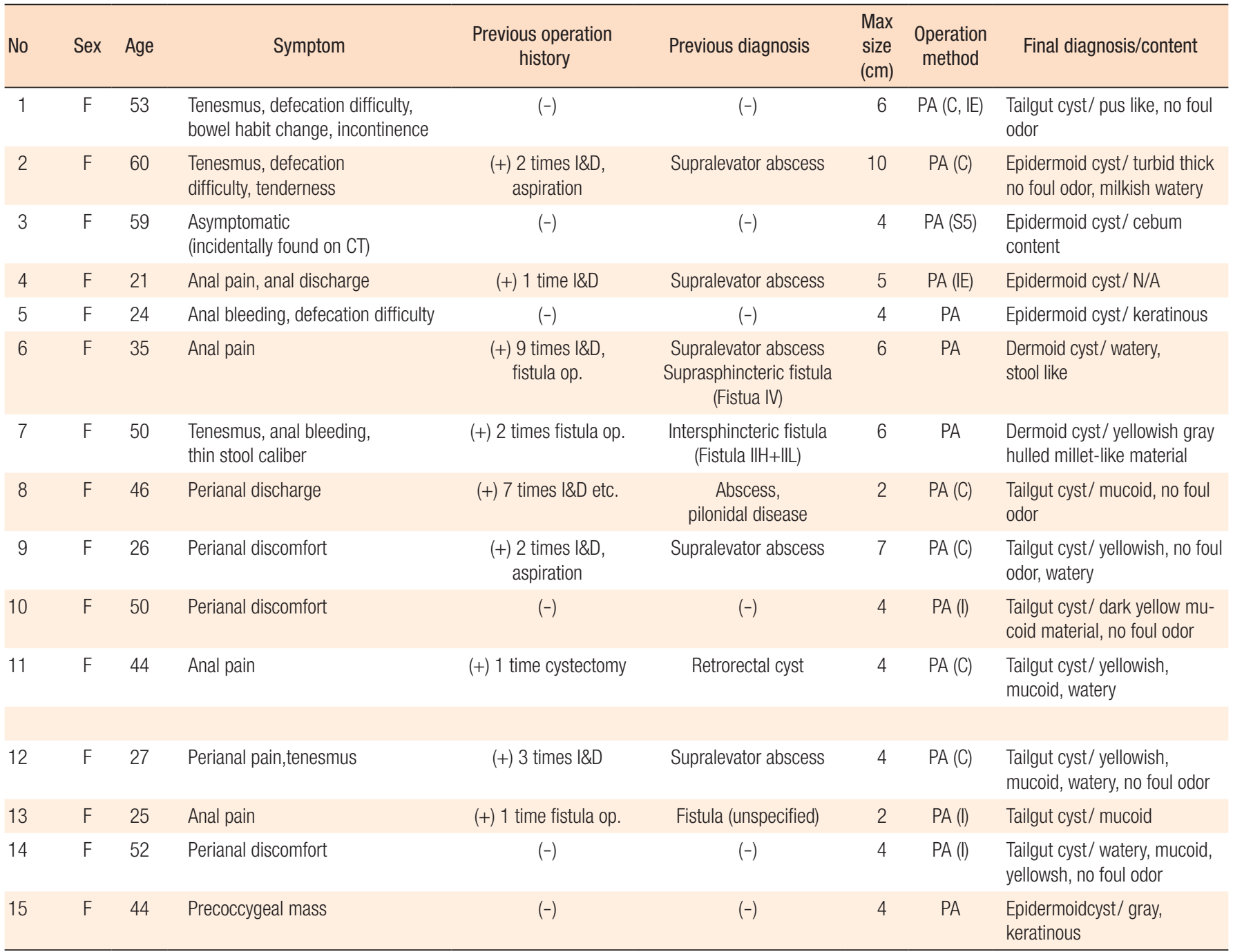

To describe the fistula type, we used both Park's and Sumikosi's classifications.

PA, posterior approach; C, coccygectomy; IE, incomplete excision; S5, sacrectomy; I, intersphincteric approach; N/A, not available. 
Table 2. Clinical manifestation of 15 patients

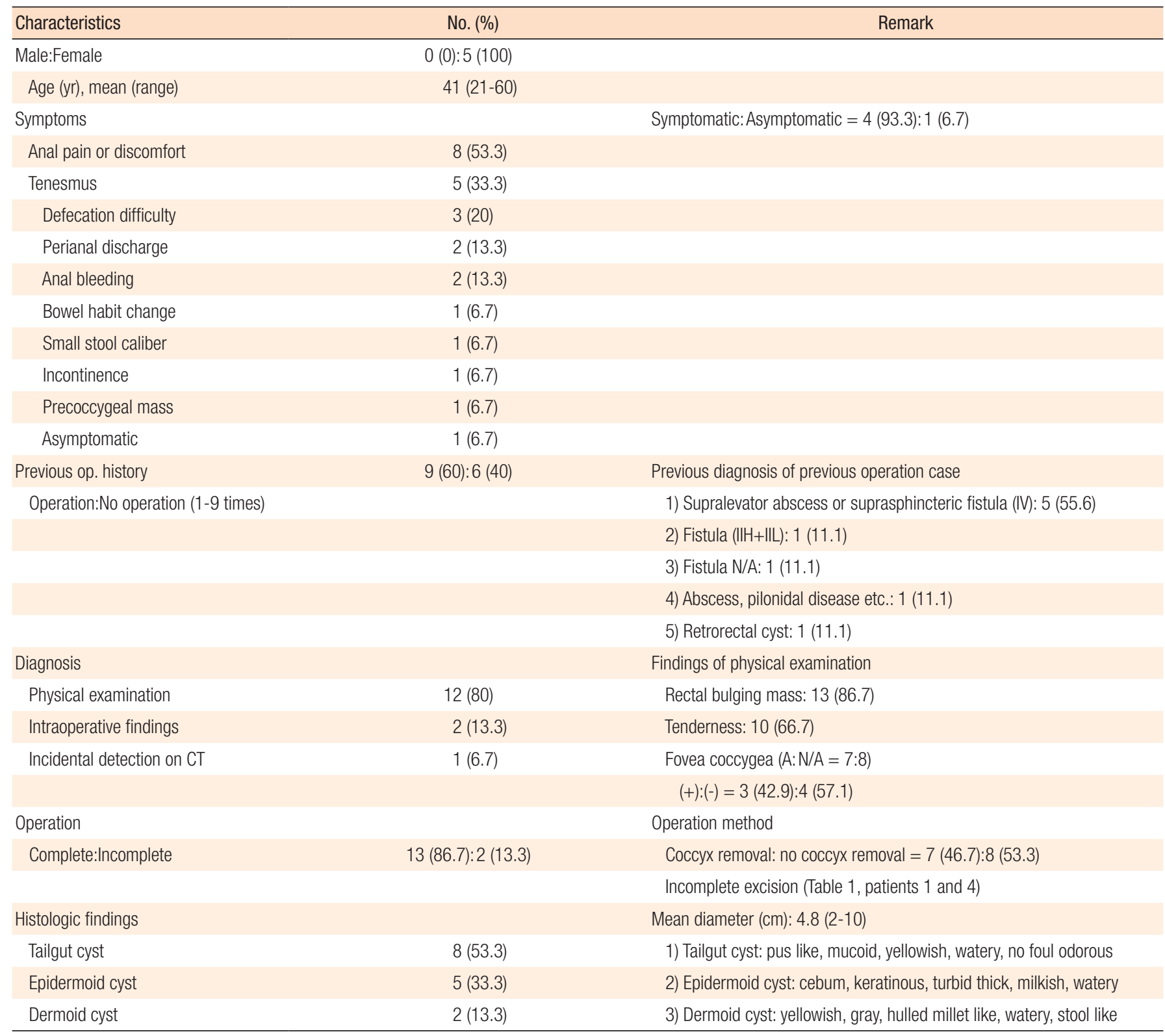

To describe the fistula type, we used both Park's and Sumikosi's classifications. A, available; N/A, not available.

(13.3\%), bowel habit changes (6.7\%), thin stool caliber (6.7\%), fecal incontinence (6.7\%), and a sacrococcygeal lump (6.7\%).

Of the 15 patients, 9 had undergone one or more operations (1-9 times) prior to the diagnosis in this hospital, and 5 of the $9(55.6 \%)$ had operations after having been diagnosed with a supralevator abscess, a suprasphincteric fistula in Park's classification (IV type in the Sumikosi classification). Three other patients were diagnosed, respectively, with an intersphincteric fistula (IIH+IIL type in Sumikosi classification, 11.1\%), an unspecified fistula (11.1\%), and pilonidal disease or perianal abscess (11.1\%). The last patient
(11.1\%) was diagnosed with a retrorectal cyst at another hospital, but it was a recurrent case (11th patient in Table 1).

In 2 of the 15 patients (13.3\%) (4th and 8th patients in Table 1), the diagnosis of a retrorectal cyst was made during the operation for a fistula and a perianal abscess and in 1 case (6.7\%) (3rd patient in Table 1), the diagnosis was made accidently on a CT scan during health screening. Apart from these, the diagnoses of the other 12 cases $(80 \%)$ were made by history taking and physical assessment and were confirmed by CT or magnetic resonance imaging (MRI). In 3 cases (20\%), including 2 cases diagnosed during the 

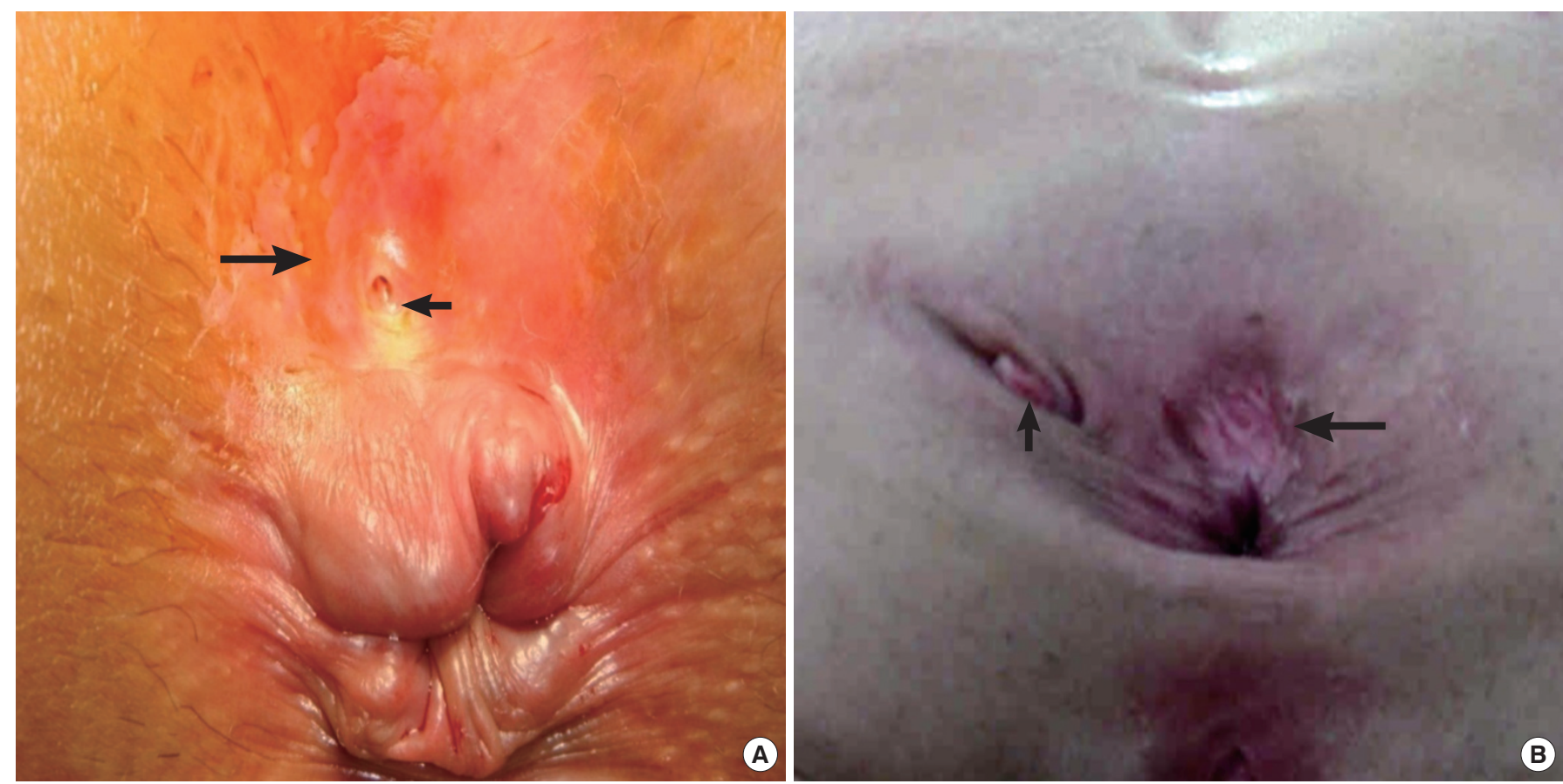

Fig. 1. (A) Funnel-shaped skin dimple (big arrow) and opening (small arrow) were noticed on the posterior anal area. The opening seems to have a 'congenital look. (B) In the midline of the posterior anal area, a funnel-shaped skin dimple (big arrow) was noticed. An opening which was made by previous drainage (small arrow), was noticed on the left lateral side of the anus. The opening has an 'acquired look'.

operation, even though the patients had undergone MRI prior to the diagnosis, they were initially misdiagnosed as having a complicated fistula and a supralevator abscess, but the diagnoses were later corrected (4th, 6th, and 8th patients in Table 1).

A soft prominence was palpable in 13 patients (86.7\%) during the physical examination and 10 patients (66.7\%) complained of tenderness in the retrorectal area. The existence of a posterior anal funnel-shaped dimple (Fovea coccygea) (Fig. 1) was investigated in 7 patients and was confirmed in 3 patients (42.9\%), but not in the other $4(57.1 \%)$. We were unable to confirm the existence or absence of a posterior anal funnel-shaped dimple in the other 8 cases.

One of the patients (12th patient in Table 1) (6.7\%) presented with a mild fever of around $37.5^{\circ} \mathrm{C}$ and increased leukocytes of 11,000; the other 14 patients (93.3\%) were apyrexial and had normal levels of leukocytes. A posterior approach was used in all surgeries. A coccygectomy was done in 7 cases (46.7\%), coccyx was not removed in the other 8 cases (53.3\%) , and intersphincter approach was used in 3 (20\%) among this 8 cases. A total excision was done in 13 cases (86.7\%); the 2 cases (13.3\%) (1st and 4 th patients in Table 1), which had fecal incontinence prior to the surgery and a high risk of worsening fecal incontinence, did not undergo a total excision. The maximum diameter of the cyst was $4.8 \mathrm{~cm}$ on average (range, 2 to $10 \mathrm{~cm}$ ). Histological investigation revealed 8 tailgut cysts (53.3\%), 5 epidermal cysts (33.3\%) and 2 dermoid cysts (13.3\%); there were no malignancies. The mean follow-up period was 18.3 months (range, 1 to 64 months), and wound infection occurred in 1 case (12th patient in Table 1), but was resolved with conservative management and did not recur.

\section{DISCUSSION}

Retrorectal or presacral tumors are extremely rare diseases. Whittaker and Pemberton reported 22 cases in the Mayo clinic during the 15 years period between 1922 and 1936, and Jao et al. reported 120 cases during the 20-year period between 1960 and 1979, claiming that the diagnosis was made in 1 of 40,000 registered patients [8]. Uhlig and Johnson [1] also said that the total number of reported retrorectal tumor cases in all the major hospitals in the Portland area in Oregon State for 30 years was 63, seeing on average 2 patients in the metropolitan area every year. Nationally in Korea, Kim et al. [9] experienced 15 cases over 5 years, Cho et al. [10] saw 34 cases over 5 years and 8 months and Kwon et al. [11] reported 10 cases over 6 years and claimed the diagnosis had been made on average in 1.6 cases per year. These data show retrorectal tumors to be extremely rare, but Hobson et al. [2] reported that most surgeons, even surgeons who do not work in tertiary hospitals, would have such an experience at least once in their lifetimes.

The classification by Uhlig and Johnson, which is modified from the classification by Lovelady and Dockerty, is widely used to classify retrorectal tumors as congenital, inflammatory, neurogenic, osteo and other $[1,8]$. The congenital tumor is the most common form of retrorectal tumor, and the developmental cyst, which the 
authors investigated, among the congenital tumors, is the most frequently seen. Stewart et al. [12] summarized a total of 298 retrorectal cases documented in the major literature between 1938 and 1975 (note that among the 301 cases reported by Uhlig [1], 66 were mistakenly reported as having retrorectal tumors whereas the correct number was 63), and 187 of the 298 (62\%) were congenital. A developmental cyst was found in 96 cases, of which $32 \%$ were retrorectal tumors and about $51 \%$ were congenital tumors. Glasgow et al. [13] summarized a total of 298 cases, including their own 34 cases, which had been reported in major journals between

Table 3. Cases of adult retrorectal developmental cysts reported from Korea

\begin{tabular}{|c|c|c|c|c|c|c|c|c|}
\hline No. & Reporter & Year & Sex & Age & Main symptom & $\begin{array}{l}\text { Operation } \\
\text { history }\end{array}$ & Size $(\mathrm{cm})$ & $\begin{array}{l}\text { Operation method/ } \\
\text { final diagnosis }\end{array}$ \\
\hline 1 & Kim et al. [26] & 1990 & M & 55 & Constipation & $(-)$ & $10 \times 11$ & PA/ dermoid cyst \\
\hline \multirow[t]{2}{*}{2} & Hong et al. [27] & 1990 & $\mathrm{~F}$ & 27 & Perianal mass & $(-)$ & $4 \times 4$ & PA/ epidermoid cyst \\
\hline & & & M & 43 & Constipation & $(-)$ & $15 \times 10$ & $\begin{array}{l}\text { APA (S4, 5, coccygectomy)/ } \\
\text { epidermoid cyst }\end{array}$ \\
\hline \multirow[t]{3}{*}{3} & Woo et al. [28] & 1992 & $\mathrm{~F}$ & 30 & General weakness & N/A & $6 \times 5$ & AA/ dermoid cyst \\
\hline & & & M & 55 & Constipation & $\mathrm{N} / \mathrm{A}$ & $11 \times 10$ & PA/ dermoid cyst \\
\hline & & & $F$ & 23 & Coccygeal pus discharge & N/A & $7 \times 7$ & $\mathrm{PA} /$ teratoma \\
\hline \multirow[t]{2}{*}{4} & Kim et al. [9] & 1994 & M & $\mathrm{N} / \mathrm{A}$ & N/A & $\mathrm{N} / \mathrm{A}$ & $15 \times 10$ & APA/ epidermoid cyst \\
\hline & & & $\mathrm{F}$ & & & & & PA/ epidermoid cyst \\
\hline 5 & Cho et al. [10] & 1995 & N/A & $\mathrm{N} / \mathrm{A}$ & N/A & $(+)$ & N/A & N/A/ developmental cyst: 7 cases \\
\hline \multirow[t]{2}{*}{6} & Kim et al. [29] & 1996 & $\mathrm{~N} / \mathrm{A}$ & $\mathrm{N} / \mathrm{A}$ & $\mathrm{N} / \mathrm{A}$ & N/A & N/A & PA/ teratoma: 2 cases \\
\hline & & & & & & & & PA/ dermoid cyst \\
\hline 7 & Ahn et al. [20] & 1998 & $\mathrm{~F}$ & 44 & Low abdominal discomfort & $(-)$ & $6 \times 4$ & $\begin{array}{l}\text { PA (transsphincteric incision)/ } \\
\text { tailgut cyst }\end{array}$ \\
\hline \multirow[t]{2}{*}{8} & Lee et al. [21] & 2001 & $\mathrm{~F}$ & 46 & Asymptomatic & $(-)$ & $11 \times 5.5$ & $\begin{array}{l}\text { AA (low midline incision)/ tailgut } \\
\text { cyst }\end{array}$ \\
\hline & & & $\mathrm{F}$ & 28 & $\begin{array}{l}\text { Low back pain and } \\
\text { coccygeal pain }\end{array}$ & $(-)$ & $9 \times 4$ & PA (coccygectomy)/ tailgut cyst \\
\hline 9 & Joo et al. [30] & 2003 & $\mathrm{~F}$ & 42 & Anal pain & $\mathrm{N} / \mathrm{A}$ & $9 \times 9$ & PA/ dermoid cyst \\
\hline 10 & Kim et al. [22] & 2005 & $\mathrm{~F}$ & 21 & Perianal pain, discharge & $(+)$ & $6 \times 6.5 \times 6$ & PA (coccygectomy)/ tailgut cyst \\
\hline 11 & Kang et al. [23] & 2005 & $\mathrm{~F}$ & 40 & Perianal pain & (+) Aspiration & 6 & $\begin{array}{l}\text { APA (Hartmann operation, } \\
\text { coccgectomy \& partial } \\
\text { sacrectomy)/ tailgut cyst } \\
\text { (adenoca., distant metastasis) }\end{array}$ \\
\hline 12 & Lee et al. [24] & 2007 & $\mathrm{~F}$ & 40 & Anal pain & $(-)$ & 2 & $\begin{array}{l}\text { AA (APR)/ tailgut cyst (carcinoid } \\
\text { tumor) }\end{array}$ \\
\hline 13 & Shin et al. [25] & 2008 & M & 72 & Abdominal discomfort & $(-)$ & $6 \times 5.5 \times 8$ & $\begin{array}{l}\text { AA (low midline incision)/ tailgut } \\
\text { cyst }\end{array}$ \\
\hline \multirow[t]{6}{*}{14} & Kwon et al. [11] & 2008 & $\mathrm{~F}$ & 74 & Abdominal discomfort & N/A & $10 \times 9$ & APA (APR)/ epidermoid cyst \\
\hline & & & $\mathrm{F}$ & 24 & Abdominal discomfort & & $8 \times 8$ & AA (abdominal incision)/ teratoma \\
\hline & & & $\mathrm{F}$ & 59 & Asymptomatic & & $5 \times 7$ & PA/ epidermoid cyst \\
\hline & & & $\mathrm{F}$ & 28 & Asymptomatic & & $7.5 \times 6.4$ & $\mathrm{AA} /$ rectal duplication cyst \\
\hline & & & $\mathrm{F}$ & 56 & Asymptomatic & & $6 \times 4$ & PA/ epidermoid cyst \\
\hline & & & $\mathrm{F}$ & 34 & Asymptomatic & & $6 \times 5$ & PA/ epidermoid cyst \\
\hline 15 & This report & 2009 & $\mathrm{~F}$ & $\begin{array}{c}41.0 \\
(21-60)\end{array}$ & $\begin{array}{l}\text { Symptom: Asymptomatic } \\
=14: 1\end{array}$ & $(+):(-)=9: 6$ & $4.8(2-10)$ & PA/ developmental cyst 15 cases \\
\hline Total & & & & & & & & 47 cases \\
\hline
\end{tabular}

Only retrorectal developmental cysts were selected from among the cases that were presented in the reports of No. 2, 4, 5, 6, and 8.

$\mathrm{PA}$, posterior approach; APA, anterior and posterior approach; AA, anterior approach; N/A, not available. 
1975 and 2004, and 99 cases (33\%) were found to be developmental cysts.

Generalizing reports with relatively large numbers of subjects published in the international literature to date, about 50\%-70\% of retrorectal tumors were congenital, and developmental cysts, being significant especially to surgeons, were found in about $30 \%$ $50 \%$ of all retrorectal tumors and in about $50-60 \%$ of all congenital tumors. However, it is difficult to predict the accurate prevalence of developmental cysts because there is a lack of research re- sults for large numbers of patients and because patients do not go to hospitals, about $40-50 \%$ of benign retrorectal tumors [13] and about $26-50 \%[1,3,6]$ of developmental cysts being reported to be asymptomatic. In Korea, 47 adult cases of retrorectal, presacral, or coccygeal developmental cysts were reported in surgical journals between 1990 and 2009, including the 15 cases reported in this study (Tables 3 and 4).

Surgical literature on retrorectal developmental cysts reported by Korean authors over the last 20 years on national medical jour-

Table 4. Clinical manifestations of 47 retrorectal developmental cysts in Korea adults

\begin{tabular}{|c|c|c|}
\hline Characteristics & №. $(\%)$ & Remark \\
\hline Male:Female & 1 (13.5):6.4 (86.5) & $A: N / A=37: 10$ \\
\hline \multirow[t]{8}{*}{ Age (yr), mean (range) } & $40.3(21-74)$ & $A: N / A=35: 12$ \\
\hline & & Age distribution (yr); cases (\%) \\
\hline & & 20-29: 11 (31.4) \\
\hline & & 30-39: $3(8.6)$ \\
\hline & & 40-49: 9 (25.7) \\
\hline & & 50-59: 9 (25.7) \\
\hline & & 60-69: $1(2.9)$ \\
\hline & & 70-79: 2 (5.7) \\
\hline Symptoms & & $A: N / A=35: 12$ \\
\hline Anal (or perianal) pain or discomfort & $13(37.1)$ & Symptomatic:asymptomatic = 29 (82.8):6 (17.1) \\
\hline Tenesmus & $5(14.2)$ & \\
\hline \multirow[t]{2}{*}{ Abdominal discomfort } & $4(11.4)$ & Symptoms, etc. \\
\hline & & General weakness \\
\hline \multirow[t]{2}{*}{ Constipation } & $3(8.6)$ & Sacrococcygeal mass \\
\hline & & Perianal discharge \\
\hline \multirow[t]{2}{*}{ Defecation difficulty } & $3(8.6)$ & Anal bleeding \\
\hline & & Incontinence \\
\hline Asymptomatic & $6(17.1)$ & Bowel habit change \\
\hline Other & $8(22.8)$ & Small stool caliber \\
\hline Previous op. history & & $A: N / A=25: 22$ \\
\hline Operation: No operation & $11(44): 14(56)$ & \\
\hline Operation method & & $\mathrm{A}: \mathrm{N} / \mathrm{A}=40: 7$ \\
\hline Posterior approach & $30(75)$ & \\
\hline Anterior approach & $5(12.5)$ & \\
\hline Ant. and post. approach & $5(12.5)$ & \\
\hline Histologic findings & & $A: N / A=40: 7$ \\
\hline Tailgut cyst & $15(37.5)$ & Adenocarcinoma in tailgut cyst: 1 case \\
\hline Epidermoid cyst & $13(32.5)$ & Carcinoid tumor in tailgut cyst: 1 case \\
\hline Teratoma & $7(17.5)$ & \\
\hline Dermoid cyst & $4(10)$ & \\
\hline Duplication cyst & $1(2.5)$ & \\
\hline
\end{tabular}

A, available; N/A, not available.

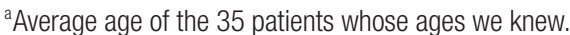


nal portal sites on the internet was reviewed by using the search terms developmental cyst, retrorectal, presacral, precoccygeal, tailgut cyst, epidermoid cyst, dermoid cyst and teratoma, and the results were summarized. Developmental cysts were reported to be 2-15 times more prevalent in women than in men and were found in all age groups, including new-born babies and infants. The sex of the patient could be identified in 37 among the 47 adult cases, and female patients were more prevalent than male patients (32 vs. 5 , respectively) with the ratio of 1:6.4 (M:F). The age could be identified in 35 cases, and the mean age was 40.3 years old (range, 21 to 74 years) and the diagnosis as made mostly in patients in their 20 's (11 cases, 31.4\%), 40's (9 cases, $25.7 \%$ ) and 50's (9 cases, $25.7 \%$ ) (Table 4). The reason for female patients being more prevalent is suspected to be the cyst's being accidentally found during regular obstetrics and gynaecology check-ups in childbearing-age women, but this suspicion was not proven $[2,4,6]$.

Of retrorectal developmental cysts, $26-50 \%[1,3,6]$ are known to be asymptomatic, and the presented symptoms are known to be similar to those of perianal inflammatory diseases, such as anorectal abscesses, anal fistulae or pilonidal disease. Consequently, colorectal specialists who examine and treat adult benign rectal diseases in their private clinics are less likely to have experience with this disease and have limited diagnostic tools in their clinics so that even if the patients present with the symptoms, a misdiagnosis as common perianal inflammatory disease could be easily made unless the possibility of the disease is kept in mind. Inappropriate surgery can damage the sphincter muscle, and subsequent fecal incontinence and structural changes, such as adhesion after surgery, make a correct diagnosis more difficult [7]. Furthermore, structural changes may make the total excision difficult, leading to serious complications such as malignancy. Hence, the first doctor who examines a patient with a developmental cyst in the retrorectal area has the best opportunity to treat the disease [7].

The presented symptoms can vary depending on the size of the cyst. The most commonly reported symptom is indescribable dull pain in the perianal area; in addition, constipation, residual sensation, changes in stool diameter, bleeding, feeling of distension in the rectum, pain on the back or pelvic area, abdominal pain and anuresis are seen. When the cyst is infected, the patient can be pyrexial [14]. A loose sphincter muscle or loss of sensation in the perineum indicates invasion to the sacral nerve [4]. The disease can also lead to difficult delivery in childbearing-age patients by blocking the parturient canal [1]. Among the 47 cases reported in Korea, the existence or the absence of any symptoms could be confirmed in 35 cases, 29 (82.8\%) being symptomatic and 6 (17.1\%) being asymptomatic, slightly less than the figures reported in the literatures $[1,3,6,13]$ (Table 4).

The most important aspect for the diagnosis is accurate physical assessment, which aids in deciding on a surgical method, as well as the diagnosis. The diagnosis of a retrorectal tumor by physical assessment alone is reported to be made in $90-100 \%$ of the patients $[2,8,15]$. The diagnosis of 12 cases among the 15 cases $(78 \%)$ in this hospital was made by history taking and physical assessment (Table 2). During the physical examination, first of all, usually a soft prominence can be felt in the retrorectal area. The finger should be rotated to examine the whole area and to evaluate the size [1]. Caution should be taken because it may feel like only mucosal folds when the cyst is relaxed. The patient might complain of tenderness, and a feeling of pushing the liquid-fulled cyst might exist. Among our 15 cases, tenderness was expressed in 10 cases (66.7\%). One of the patients (6th patient in Table 1) had had repeated surgeries after having been diagnosed as having a supralevator abscess or fistula. Even after investigations such as MRI had been performed, it was not diagnosed as a developmental cyst; the diagnosis was made retrospectively after the bubbling of gas was felt on the levator ani muscle during the follow-up. This was judged to be a collapsed cyst whose contents had been removed. Therefore, physical assessment is the single most important thing for the diagnosis.

No prominent area was felt in the retrorectal area in another case (8th patient in Table 1, Fig. 2A), and the cause of this was thought to be the cyst's being small and the contents of the cyst having been almost removed by a secondary fistula due to previous surgeries in the retrorectal area. In this case, a fistula was suspected, and a fistulectomy was performed, but the fistula was found to be connected to the presacral area during the fistulectomy. Subsequently, a precoccygeal cyst was identified after the coccyx had been removed. The diagnosis prior to surgical intervention will not be easy in cases like this.

Even if no chronic inflammation or acute infections exist, indescribable pain around the perianal area, abdominal pain, and tenderness in the retrorectal area can be induced by the pressure of a large cyst, which can be misunderstood as symptoms of a supralevator abscess. If the cyst is misdiagnosed as supralevator abscess and is drained, the cyst will collapse, and its range will be difficult to judge. Also, surgeons wait for a fistula track to be formed after incision and drainage of the cyst, but when the cyst is filled again, it might be perceived as a recurrent supralevator abscess (4th, 6th, 7th, 8th, 9th and 12th patients in Table 1). However, supralevator abscesses are more prevalent in males, and patients commonly complain of anal pain with inflammatory response, such as fever, chill and increased leukocytes, because the abscess has already progressed extensively by the time the patient comes to the hospital. Thus, especially in female patients, a possible retrorectal developmental cyst should be considered when they complain of indescribable pain in the perianal area and tenderness of a retrorectal prominence without fever, chill or increased leukocytes. In addition, it is helpful to consider a possible retrorectal developmental cyst when patients complain of a relatively low level of pain or tenderness in the retrorectal area even when the amount of pus in the physical assessment is thought to be large. Only one case (14th patient in the Table $1 ; 6.7 \%$ ) showed a mild fever of around $37.5^{\circ} \mathrm{C}$ and increased leukocytes (around 11,000) and was suspected of having an infection during the diagnosis of a retrorectal developmental cyst in this hospital. However, in the other patients, there were no 
Journal of The Korean Society of Clinical Study and Review of Articles (Korean) about Retrorectal Developmental Cysts in Adults

Coloproctology sung Wook Baek, et al.
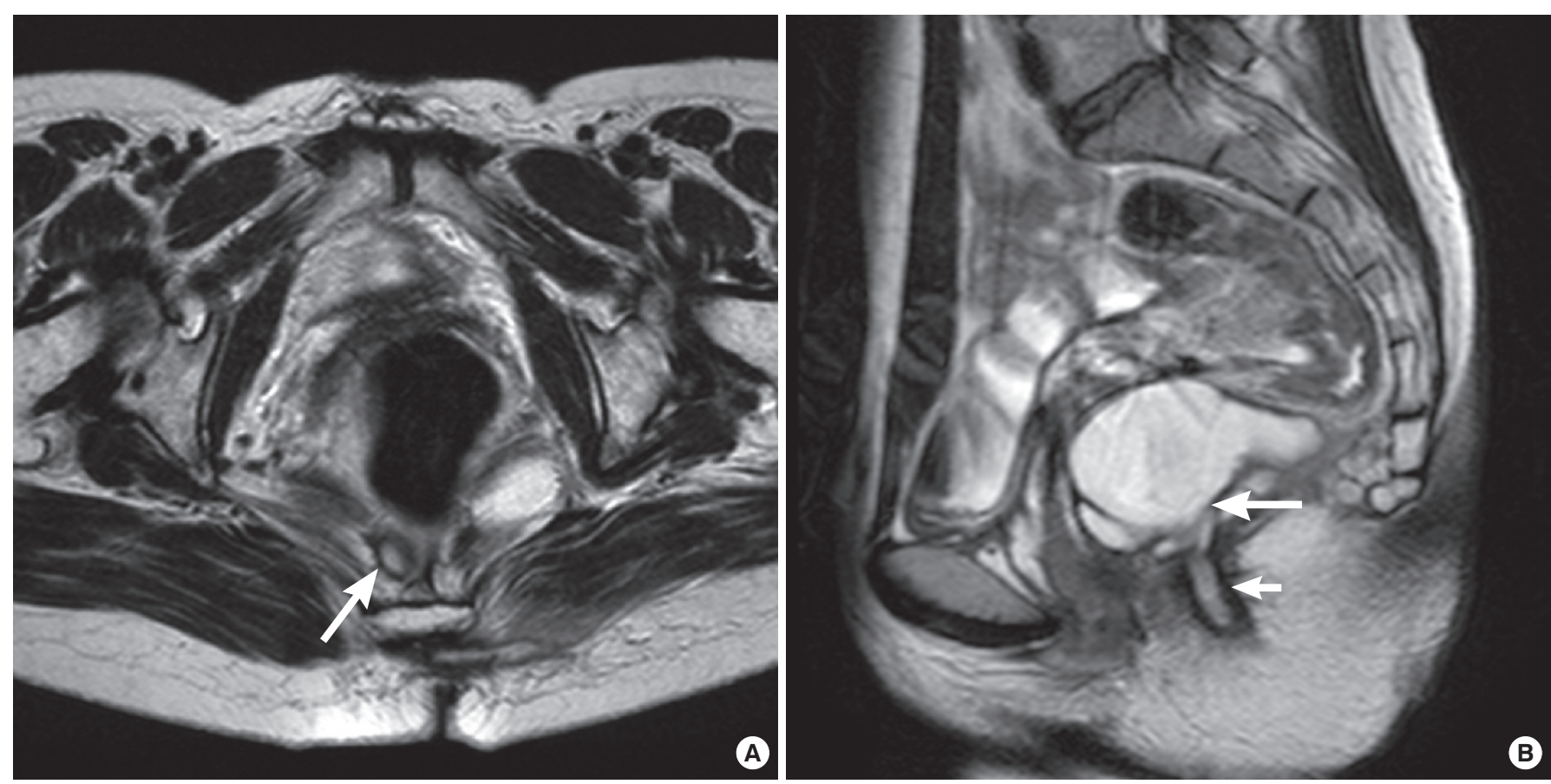

Fig. 2. (A) On magnetic resonance imaging (MRI; T2WI), a small lesion with a high signal density (arrow) was noted on the retrorectal area. The lesion was so small that it was preoperatively diagnosed as an anal fistula, but intraoperatively it was diagnosed as a developmental cyst. (B) On the MRI (T2WI), a multiloculated cystic lesion (big arrow) with a high signal density was noticed in the retrorectal area, and a secondary opening (small arrow) made by a previous operation was noticed (same patient as the one in Fig. 1B).

signs of infection, such as fever, chill or increased leukocytes, even though the patients complained of pain or tenderness in the retrorectal prominence or the perianal area.

Pilonidal disease is prevalent in hairy male patients, and a lump is not palpable in the retrorectal area in the disease, so the cyst can be diagnosed. Secondarily, the existence or absence of a characteristic posterior anal funnel-shaped dimple (=fovea coccygea) and opening on the dimple in the midline, which was caused by a connection between the retrorectal developmental cyst and skin (Fig. 1), should be confirmed. These kinds of retrorectal excavations have been reported to have various rates of occurrence of about 30-100\% in the literature $[1,2,16]$. Grandjean et al. [3] reported these findings as being typical of tailgut cysts. About $30-50 \%$ of developmental cysts are reported to be chronically infected $[4,5]$, so caution should be taken because perianal pain and tenderness caused by inflammation of a developmental cyst can lead to a midline opening on the dimple being misunderstood as the secondary opening of a common perianal fistula. The opening of a developmental cyst has a 'congenital and dimpled look', but the opening secondary to a perianal fistula has an 'acquired look' (Fig. 1). The secondary opening usually develops from an infected anal gland and can be distinguished from the congenital opening of a developmental cyst because the secondary opening of a perianal fistula usually exists in the same level of the skin's surface, can develop in a non-specific area, such as the perianal area, perineum or the gluteal area, and can be accompanied by granulation tissues [17]. However, if the developmental cyst is initially misdiagnosed and the original posterior anal dimple and midline opening are removed, these lesions will be much less probable to be confirmed in later surgery, and the developmental cyst might be repeatedly misdiagnosed.

If there is a perianal scar due to pervious repeated surgery, a developmental cyst should be suspected. This is important because if the cyst had been misdiagnosed as a perianal fistula or a supralevator abscess by the first doctor who performed a surgery, the next doctor might diagnose it as a recurring abscess because of preconception. The surgical histories of 25 of the 47 cases reported in Korea could be confirmed. Eleven of them (44\%) had more than one surgery for the same lesion (Table 4).

Hawkins and Jackaman [16] pointed out that a retrorectal developmental cyst should be suspected especially when female patients come to the hospital presenting histories of recurrent anal or rectal abscess and fistula, history of repeated surgery for these lesions, a fistula with a posterior anal funnel-shaped dimple even if it is not infected, a palpable lump in the precoccygeal or the prescral area, hairy or cheesy discharge from the anus or a perianal fistula. Spencer and Jackaman [17] concluded that a congenital developmental cyst should be suspected in patients with a recurrent retrorectal abscess, a repeated fistulectomy, presence of a fistula in the anus or the perianal area or rectum without identification of a primary focus in the dentate line of the anus, a posterior anal funnel- 
shaped skin dimple and palpable fixed or distended lesion in the precoccygeal area.

In terms of diagnostic investigation, the size of tumor, the cyst structure, the level of invasion to rectum, and lymphatic metastasis, if the cyst becomes malignant, can be confirmed by using transrectal ultrasonography [13]. With endoscopy, prominent mucosa in retrorectal area can be seen if the lesion is large enough, and the level of proximal extension can be confirmed as well [2]. A thin external wall and cystic tumor with septation can be seen on CT. With CT, malignancy should be suspected if there is a calcified cystic wall, and this calcification is more common in a dermoid cyst or a teratoma. Rarely can air inside the cyst be seen when a fistula is formed.

MRI has become the most important investigative tool in recent years, replacing the other radiological examinations. MRI provides the location, the size and the characteristics of the tumor precisely, as well as vital information for aiding the decision on surgical treatment. In general, developmental cystis recognized as a cyst with low signal intensity and a distinctive thin external layer in theT1 weighted image and as a cysts with high signal intensity in the T2 weighted image. However, they can be recognized as having a high signal intensity even in the $\mathrm{T} 1$ weighted image if the contents of cyst are mucoid (tailgut cyst) or fatty (dermatoid cyst) (Fig. 2B). Even in the T1 weighted image, tailgut cysts can be recognized variously as having low to high signal intensity depending on the density and the viscosity of the mucinous material and the high-protein components and on the presence or absence of bleeding inside the cyst. The T2 weighted image is more useful to confirm smaller cysts and septation than the T1 weighted image or the CT image. Whereas other types of developmental cysts are monolocular, tailgut cysts usually have a multilocular structure or the structure of small cysts attaching to a bigger main cyst, so a different signal intensity can be seen in each locule of a multilocular cyst [18]. The cystic wall will become thicker when a developmental cyst is infected, and the borderline of the wall will become less clear as it progresses to malignancy [11]. Therefore, malignancy can be suspected if the wall of a cyst has an irregular, thickened look on the T1 and T2 images or on the contrast image. However, a comprehensive approach that considers medical history, symptoms and physical assessment, is important because a developmental cyst still can be misdiagnosed as a supralevator abscess or a complicated anal fistula despite a preoperative MRI (4th, 6th, and 8th patients in Table 1).

Biopsy prior to an operation is usually not recommended because an accurate pathological diagnosis with a local biopsy is often impossible and because the biopsy can cause secondary infection or spread of cancer cells if the cyst is malignant. Therefore, a biopsy should be performed selectively for conservative management such as radiotherapy when sacral invasion of a malignancy that is not feasible to remove is suspected. Also, a biopsy via the rectum should be avoided to prevent cancer cells from spreading into the rectum, and a CT-guided extra-rectal approach or presacral approach is recommended $[2,4]$.

Differential diagnoses that need to be eliminated are anal fistula, perianal abscess, pilonidal disease, other types of retrorectal congenital tumors, neurogenic tumors and osseous tumors. Consultation with a neurosurgeon or an orthopedic surgeon is essential if these diseases are suspected.

The final pathological diagnosis and treatment can be done by removing the cyst completely. The retrorectal space is a potential area that appears only when the rectum is displaced in the anterior direction by a tumor and is the tumor borders the rectum in the anterior direction, the sacrum and the coccyx in the posterior direction, the peritoneal reflection in the superior direction, the levator ani muscle and the coccygeal muscle in the inferior direction, and the iliac vessels and the ureters on the left and the right side. In the retrorectal space are the sacral plexus, middle hemorrhoidal vessels, median sacral vessels and lymph nodes. This space can be generally approached with an anterior approach, a posterior approach and an anterior-posterior approach, depending on the size and the location of a tumor. There are differences in authors' opinions, but the posterior approach via the anus and perineum should be attempted when the size of a tumor is less than $5 \mathrm{~cm}$ and the whole tumor feels as if it is located in a relatively inferior direction. In the posterior approach, a Kraske operation and a paracoccygeal approach, which access a tumor after incising posterior to the anus and removing the coccyx, are usually used, but an intersphincteric approach, which accesses a tumor after opening the intersphincteric space and dividing the levator ani muscle, can also be used. Operational methods could be confirmed in 40 of the 47 Korean cases, and the posterior approach was used in 30 of those 40 (75\%) (Tables 3 and 4). A conventional coccygectomy has been reported to be vital in securing enough visual field for complete excision during a posterior approach when severe adhesion to surrounding tissues caused by repeated operations or infection exists [2]. In this hospital, a posterior approach using a coccygectomy was used in 7 of our 15 cases (46.7\%) (Fig. 3).

When the cyst is adhered to the coccyx or becomes malignant and invades the coccyx, the residual cells can cause recurrence. Neoplastic cells are reported to develop easily in the coccyx; hence, a coccygectomy is essential to cure the disease, especially in patients with a teratoma [2]. A partial sacrectomy might be necessary in the case of malignant tumor invasion to the sacrum or be needed to secure a visual field (3rd patient in Table 1). A partial sacrectomy from under the S3 border can be performed without damaging the function of the anal sphincter. Removal above the S3 nerve root bilaterally can cause fecal incontinence, urinary incontinence and impotence $[13,19]$.

Complete cyst removal was impossible in 2 of our 15 cases (13.3 $\%)$. The first case had a high risk of rectal perforation and already had relatively severe fecal incontinence prior to the surgery. The other case had a high risk of worsening fecal incontinence due to reduced anal pressure on anal manometry and unilateral delayed latency on pudendal nerve terminal motor latency (PNTML) prior 
Journal of The Korean Society of Clinical Study and Review of Articles (Korean) about Retrorectal Developmental Cysts in Adults

\section{Coloproctology sung Wook Baek, et al.}
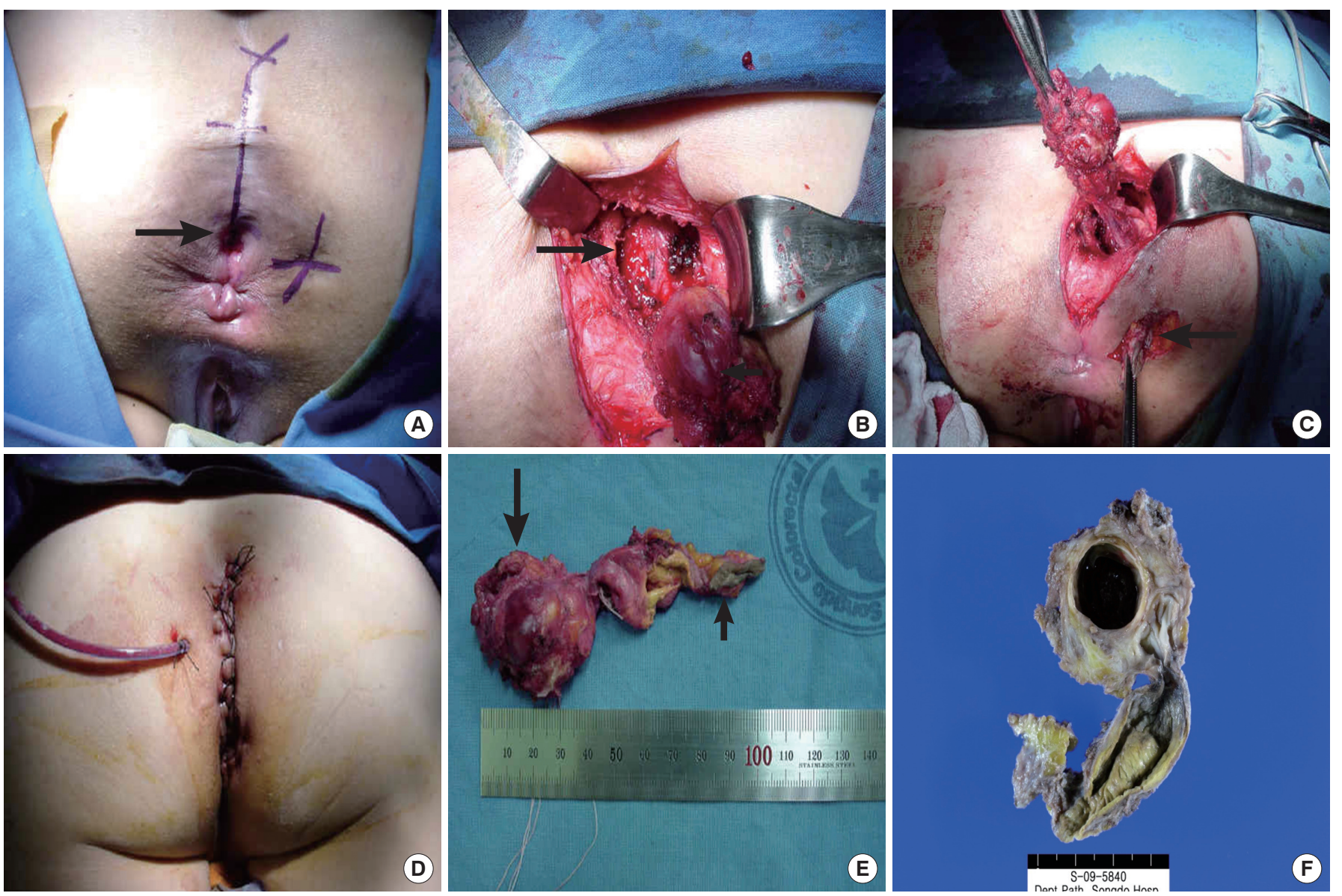

Fig. 3. (A) On the right side of the anus, an iatrogenic fistula opening from a previous operation was noticed. On the posterior side, a funnelshaped dimple (arrow) was noticed. An artificial line was drawn preoperatively for incision from the anal verge to the coccyx. (B) Coccyx was removed. After excision of the cyst (small arrow), a retrorectal space (big arrow) was exposed. (C) An iatrogenic secondary tract (arrow) that was made after a previous operation was excised. (D) A closed drainage catheter was inserted, and the wound was closed with interrupt sutures. (E) Excised cyst (big arrow) and secondary tract (small arrow) made by a previous operation. (F) A multiloculated cystic structure was noticed after dividing the specimen fixed with formalin.

to the surgery (1st and 4th patients in Table 1).

Intersphincteric approach causes less damage to the surrounding tissues, enables to preserve the function of anal sphincter, prevents unnecessary damage to sacral nerve and induces less complications, such as urinary retention, because the intersphincteric space is embryologically avascular. However, it is only applicable to small cysts (10th, 13th, and 14th patients in Table 1).

An anterior approach via the abdomen is recommended when the size of a tumor is about $5 \mathrm{~cm}$ and the upper border of the tumor is located above the 3rd sacrum and the lower border of the tumor does not reach the 4th sacrum whereas an anterior-posterior approach is recommended if a tumor is bigger than $5 \mathrm{~cm}$ and is formed largely across the sacrum or malignancy is suspected or severe adhesion exists [2-4, 13]. However, a cyst can be removed completely with a posterior approach after the contents have been drained carefully even when the cyst is large as long as there is no evidence of infection or adhesion (1st and 9th patients in Table 1). A laparoscopic anterior approach can also be used safely under the proper visual field if no evidence of malignancy exists.

Among the 47 Korean cases, histological examination results could be confirmed in 40 cases, and the cysts were mostly tailgut cysts (15 cases, $37.5 \%$ ), epidermal cysts (13 cases, $32.5 \%)$, teratomas ( 7 cases, $17.5 \%$ ), dermoid cysts ( 4 cases, $10 \%$ ), rectal intussusceptions (1 case, 2.5\%) (Table 4). Looking at the histological characteristics of each cyst, a tailgut cyst develops when a true tail has failed in complete involution and the residual form cysts $[3,6]$. This consists of a squamous epithelium, a transitional epithelium, a glandular cilliated columnar epithelium, and a mucinous columnar epithelium because it has developed from a gastrointestinal precursor. Scattered smooth muscle fiber bundles can also be observed. It can be differentiated from a rectal intussusception which has two muscle layers that have the myenteric plexus of Auerbach 

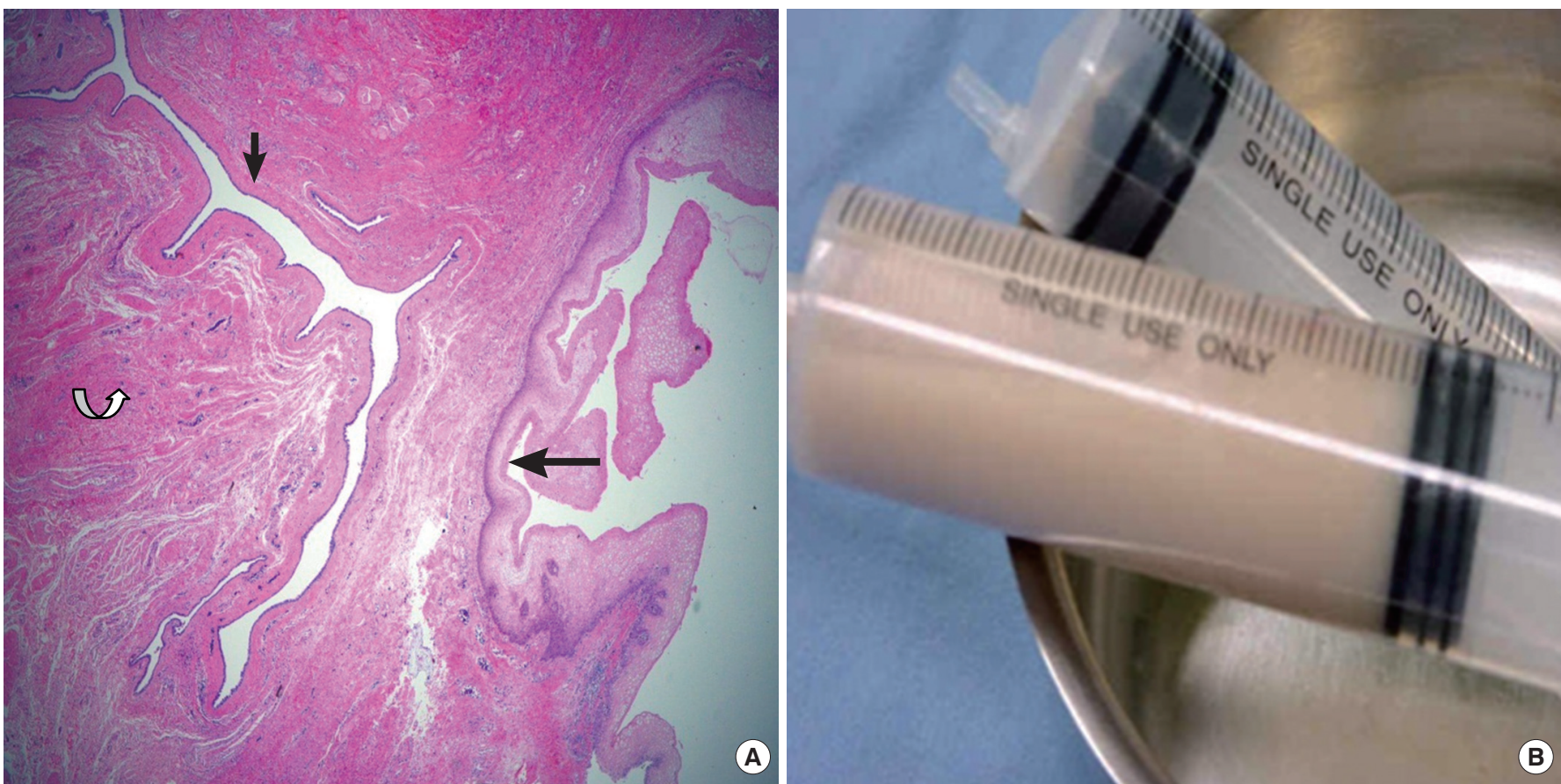

Fig. 4. (A) A multilocular structure lined with a stratified squamous epithelium (big arrow) and a columnar epithelium (small arrow) is noticed. A smooth muscle bundle (curved arrow) was also found. (H\&E, $\times 20)$. (B) The content of tailgut cyst was somewhat yellowish and watery. It was different from the conventional foul odorous creamy pus composed of liquefied material of fat tissue.

[20-24] (Fig. 4A). An epidermal cyst can develop due to closing a defect of the ectodermal tube; consequently, it is covered only with a squamous epithelium. The genesis of a dermoid cyst is similar to that of an epitheloid cyst, but the cystic wall includes mature dermoid adnexals, such as a sebaceous gland, a pilar cyst or a sudoriferous gland, as well as a squamous epithelium. A teratoma can have any type of tissue originating from three kinds of embryonic germ cells as it is developed from a totipotential cell [2]. Although a teratoma is usually found in children, it can be found in adulthood if it is too small or the symptoms are extremely mild. A rectal intussusception is connected with the rectum and includes structures such as mucosa with lamina muscularis mucosae, muscle layers, serosa, villous and crypt, as well as two clear muscle layers with the myenteric plexus of Auerbach [3].

Investigating the cyst's contents is essential because even if it is misdiagnosed as a supralevator abscess and an incision and drain is performed, repetitive misdiagnosis can be prevented as long as the contents have been investigated. The contents of a tailgut cyst do not have a foul odor unless it is infected, and in a tailgut cyst, cloudy clear, yellow, green or brown substances, including mucin, can be identified. The contents of tailgut cyst will be somewhat watery due to the large mucinous content, and even when it is infected, the contents will be distinguishable from the usual creamy foul odorous pus generated by liquefaction of fatty tissue around the anorectal area because the major content is mucin. The contents of the tailgut cysts excised at this hospital were watery and light yellow without foul odor (Table 1, Fig. 4B). The contents of epidermal cysts and dermoid cysts are smooth, cheesy-type substances and the contents can turn into pus when is the cysts are infected. Therefore, when the content looks like a smooth, cheesy discharge combined with pus, these types of cysts should be suspected.

In a tailgut cyst, depending on the cells lining the cyst, it can develop into a malignant squamous cell carcinoma, an adenocarcinoma or a carcinoid tumor. One case of an adenocarcinoma and one case of a carcinoid tumor among tailgut cyst were reported in Korea $[23,24]$. The malignant change process of an adenoma is assumed to be related to a p53 gene mutation, similar to that of the dysplasia-adenocarcinoma sequence in colon cancer.

As a tumor marker, increased alpha-fetoprotein (AFP) and carcinoembryonic antigen (CEA) can be found in a teratoma and they are used in follow-up investigation. Increased CEA can be identified in tailgut cysts. Observations that tumor markers are increased in malignant tailgut cysts and decreased down after the excision indicate that a connection between these tumor markers and malignancy is probable, although these tumor markers do not always reflect malignancy $[23,25]$.

A squamous cell carcinoma can develop in an epidermal cyst. A teratoma is usually benign in new-born babies, but the cyst has a higher risk of becoming malignant as a baby becomes older, and the cyst contains more solid components. A teratoma is also usually benign in adult patients, but $5-10 \%$ of teratomas become malignant if they are not cured [2]. 
Malignancy was not identified in the patient (11th patient in Table 1) who underwent reoperations due to recurrence. The authors followed the patients with endorectal ultrasonography and MRI, and the average follow-up period was 18.3 months (range, 1 to 54 months).

Strong suspicion and physical assessment are the most important factors in diagnosing a retrorectal developmental cyst. The presence of a retrorectal developmental cyst should also be borne in mind when a proctologist examines adult female patients with a history of repeated surgery for an anal fistula, a soft retrorectal prominence, a funnel-shaped posterior anal dimple, an external opening in the retrorectal area without fever or chill, and a discharge that is somewhat watery, unlike the usual foul odorous creamy pus when a cyst is drained. Research with various examples is required in the future.

\section{CONFLICT OF INTEREST}

No potential conflict of interest relevant to this article was reported.

\section{REFERENCES}

1. Uhlig BE, Johnson RL. Presacral tumors and cysts in adults. Dis Colon Rectum 1975;18:581-9.

2. Hobson KG, Ghaemmaghami V, Roe JP, Goodnight JE, Khatri VP. Tumors of the retrorectal space. Dis Colon Rectum 2005;48: 1964-74.

3. Grandjean JP, Mantion GA, Guinier D, Henry L, Cherki S, Passebois L, et al. Vestigial retrorectal cystic tumors in adults: a review of 30 cases. Gastroenterol Clin Biol 2008;32:769-78.

4. Singer MA, Cintron JR, Martz JE, Schoetz DJ, Abcarian H. Retrorectal cyst: a rare tumor frequently misdiagnosed. J Am Coll Surg 2003;196:880-6.

5. Dahan H, Arrive L, Wendum D, Docou le Pointe H, Djouhri H, Tubiana JM. Retrorectal developmental cysts in adults: clinical and radiologic-histopathologic review, differential diagnosis, and treatment. Radiographics 2001;21:575-84.

6. Hjermstad BM, Helwig EB. Tailgut cysts: report of 53 cases. Am J Clin Pathol 1988;89:139-47.

7. Theuerkauf FJ Jr, Hill JR, ReMine WH. Presacral developmental cysts in mother and daughter: report of cases. Dis Colon Rectum 1970;13:127-32.

8. Jao SW, Beart RW Jr, Spencer RJ, Reiman HM, Ilstrup DM. Retrorectal tumors: Mayo Clinic experience, 1960-1979. Dis Colon Rectum 1985;28:644-52.

9. Kim JI, Hong KH, Joo JS, Kim BH. A clinical study of retrorectal tumos. J Korean Soc Coloproctol 1994;10:195-9.

10. Cho YP, Kim JC, Kim YT, Lim SC, Park KC. Retrorectal presacral tumor. J Korean Soc Coloproctol 1995;11:165-72.

11. Kwon HY, Hur H, Min BS, Kim NK, Sohn SK, Cho CH. Clinico- pathological features of retrorectal tumors in an adult: a case report and review of the literatures. J Korean Soc Coloproctol 2008; 24:292-7.

12. Stewart RJ, Humphreys WG, Parks TG. The presentation and management of presacral tumours. Br J Surg 1986;73:153-5.

13. Glasgow SC, Birnbaum EH, Lowney JK, Fleshman JW, Kodner IJ, Mutch DG, et al. Retrorectal tumors: a diagnostic and therapeutic challenge. Dis Colon Rectum 2005;48:1581-7.

14. Gonul II, Baglan T, Pala I, Mentes B. Tailgut cysts: diagnostic challenge for both pathologists and clinicians. Int J Colorectal Dis 2007;22:1283-5.

15. Lev-Chelouche D, Gutman M, Goldman G, Even-Sapir E, Meller I, Issakov J, et al. Presacral tumors: a practical classification and treatment of a unique and heterogeneous group of diseases. Surgery 2003;133:473-8.

16. Hawkins WJ, Jackman RJ. Developmental cysts as a source of perianal abscesses, sinuses and fistulas. Am J Surg 1953;86:678-83.

17. Spencer RJ, Jackman RJ. Surgical management of precoccygeal cysts. Surg Gynecol Obstet 1962;115:449-52.

18. Aflalo-Hazan V, Rousset P, Mourra N, Lewin M, Azizi L, Hoeffel C. Tailgut cysts: MRI findings. EurRadiol 2008;18:2586-93.

19. Losanoff JE, Sauter ER. Retrorectal cysts. J Am Coll Surg 2003; 197:879-80.

20. Ahn BY, Jeong CS, Lee DH, Yu CS, Lee HJ, Lee MK, et al. Tailgut cyst: a case report. J Korean Soc Coloproctol 1998;14:617-20.

21. Lee H, Oh JH, Cho SY, Yang DM, Ha SY. Two cases of tailgut cyst. J Korean Soc Coloproctol 2001;17:209-12.

22. Kim SS, Kim JY, Lee HK, Hwangbo S, Kim JG, Lee DH, et al. A case of a tailgut cyst. J Korean Soc Coloproctol 2005;21:105-8.

23. Kang SW, Kim NK, Baik SH, Lee KY, Sohn SK, Cho CH. Adenocarcinoma arising from tailgut cyst. J Korean Surg Soc 2005;68: 342-5.

24. Lee CM, Lee SH, Jeon CW, Ahn BK, Baek SU. Carcinoid tumor arising within a tailgut cyst: a case report. J Korean Soc Coloproctol 2007;23:65-7.

25. Shin IY, Lee IK, Lee YS, Kim DH, Oh ST, Kim JG, et al. The diagnosis of tailgut cyst in the setting of elevated serum CEA levels. J Korean Surg Soc 2008;75:272-6.

26. Kim TS, Kim JP. Presacral epidermal inclusion cyst. J Korean Surg Soc 1990;39:140-4.

27. Hong KH. Posterior approaches for rectal and retrorectal diseases. Inje Med J 1990:11:125-9.

28. Woo YM, Jung YK, Park JG, Choi KJ, Kim JP. Surgical management for sacral and presacral tumor. Cancer Res Treat 1992;24: 443-9.

29. Kim JM, Kim HD, Kim KY. Surgical approach to tumor of rectum and retrotectal space: posterior rectotomy. J Korean Soc Coloproctol 1996;12:127-35.

30. Joo JK, Ryu SY, Kim DY, Kim YJ, Lee JH. Dermoid cyst in the ischiorectal space. J Korean Soc Coloproctol 2003;19:391-3. 